\title{
An "Ageing" Operator and Its Use in the Highly Constrained Topological Optimization of HVAC System Design
}

\author{
Jonathan Wight, Yi Zhang \\ Department of Civil and Building Engineering, Loughborough University, \\ Loughborough, Leicestershire, \\ LE11 3TU, UK \\ Tel: +44(0)1509-620365 \\ J.A.Wright@lboro.ac.uk
}

\begin{abstract}
The synthesis of novel heating, ventilating, and air-conditioning (HVAC), system configurations is a mixed-integer, non-linear, highly constrained, multi-modal, optimization problem, with many of the constraints being subject to time-varying boundary conditions on the system operation. It was observed that the highly constrained nature of the problem resulted in the dominance of the search by a single topology. This paper, introduces an new evolutionary algorithm operator that prevents topology dominance by penalizing solutions that have a dominant topology.
\end{abstract}

The operator results in a range of dynamic behavior for the rates of growth in topology dominance. Similarly, the application of the ageing penalty can result in the attenuation of topology dominance, or more severely, the complete removal of a topology from the search. It was also observed that following the penalization of a dominant topology, the search was dynamically re-seeded with both new and previously evaluated topologies. It is concluded that the operator prevents topology dominance and increases the exploratory power of the algorithm.

The application of an evolutionary algorithm with ageing to the synthesis of HVAC system configurations resulted in a novel design solution having a $15 \%$ lower energy use than the best of conventional system designs.

\section{Categories and Subject Descriptors}

I.2.8 [Artificial Intelligence]: Problem Solving, Control Methods, and Search - heuristic methods.

J.6 [Computer-Aided Engineering]: Computer-aided Design (CAD).

General Terms: Algorithms, Design.

Keywords: Evolutionary Algorithms, Topological Optimization, HVAC, System Design.

\footnotetext{
Permission to make digital or hard copies of all or part of this work for personal or classroom use is granted without fee provided that copies are not made or distributed for profit or commercial advantage and that copies bear this notice and the full citation on the first page. To copy otherwise, or republish, to post on servers or to redistribute to lists, requires prior specific permission and/or a fee.

GECCO'05, June 25-29, 2005, Washington, DC, USA.

Copyright 2005 ACM 1-59593-010-8/05/0006 ...\$5.00.
}

\section{INTRODUCTION}

The temperature and humidity of the air in the occupied spaces of commercial buildings, is maintained by "heating, ventilating and air conditioning" (HVAC), systems. HVAC systems ventilate buildings by taking in outside air and mixing it with air that has been re-circulated from within the building. The ventilation air is further conditioned by heating, cooling, and humidifying components. The ventilation air maintains the room temperature and humidity by being supplied to the room at a condition that offsets thermal loads on the room.

The air conditioning components can be connected together in a number of ways to produce a viable system configuration. Over the last 100 years, a number of recognized configurations have evolved through the process of heuristic design [2]. The alternative design solutions have been driven predominantly by the need to limit the capital cost of the systems. This has led to system configurations that are able to maintain the temperature and humidity in more than one "control zone" (a control zone being a collection of rooms that experience similar heat loads). However, many of the established "multi-zone" systems have a higher than necessary energy consumption (a "multi-zone" system is one that simultaneously conditions more than one control zone).

Concerns over climate change and associated energy use has renewed interest in the design of HVAC systems for low energy use. This paper describe an approach to the automatic synthesis of HVAC system configurations for minimum energy use by an evolutionary algorithm. A new algorithm operator which is designed to maintain the power of the search in exploring alternative system topologies is also described.

\subsection{Problem Characteristics and Optimization Approach}

There are three elements to the design of an HVAC system,

1. the selection of a component set (the choice of type and number of components);

2. given the component set, the design of the feasible topology;

3. optimization of the system operation (for several different operating conditions).

The selection of a feasible topology is dependent on the component set and similarly, the optimization of system operation 
on the configuration. Hence, conventionally, the optimization of such systems is conducted in a iterative manner, the iteration sequence being first, the selection of a component set, followed by the optimization of the system topology (with the evaluation of each trail topology requiring the optimization of the system operation). However, the need for repeated optimization of the system topology and operation, results in the iteration being computational intensive. Hence, the approach adopted here $[1,7]$, is that all three elements of the design problem are optimized simultaneously. In the experiments described here, however, the component set has been fixed so that the optimization is concerned only with the choice of topology and system operation.

Note that HVAC systems are subject to time varying boundary conditions (namely, the ambient climate and heat gains arising inside the building), and that the system operation must be optimized at each boundary condition. In the work reported here, the system operation has optimized for 9 boundary conditions that represent a period during the early morning, mid-day, and afternoon, in each of three seasons (winter, spring or fall, and summer).

The optimization problem can be described as a mixed-integer, non-linear programming problem, having non-linear inequality and equality constraints. The problem is also highly multi-modal in that there are many alternative system configurations, each resulting in a local optimal solution for system operation. The decision variables are derived from the representation of the system topology, and the system operation (operation being optimized for all 9 condition simultaneously). The problem constraints maintain the feasibility of the topology and the system operation. Finally, the objective function is formed from the annual system energy use as estimated from the 9 operating conditions.

The general approach to the problem formulation and solution has been described previously [1,7], and is therefore only summarized here.

\subsubsection{Decision Variables and Encoding}

There are two sets of decision variables, those relating to system topology $X_{d}$, and those to the system operation, $X_{c}$. The set of decision variable $X$, can be defined by:

$$
X=\left(X_{d}, X_{c}\right)
$$

where:

$$
\begin{aligned}
& X_{d}=\left(x_{d}, \ldots . ., x_{d, n d}\right) \in Z^{n d} \\
& X_{c}=\left(x_{c}, \ldots ., x_{c, n c}\right) \in R^{n c}
\end{aligned}
$$

Each set of variables has been encoded in a separate chromosome, the topology variables as an integer chromosome, and the system operation variables as a real vector chromosome. The system topology is represented by a modified adjacency matrix (system graph), that has been designed to limit the duplicate representation of topologies (although it does not eliminate this effect completely). The topology chromosome has also been designed to facilitate the implementation of problem specific recombination and mutation operators $[1,7]$.

The system operation variables are in two groups, those that define the air-flow rate through the system and those that define the thermal capacity (output) of the system components.

\subsubsection{Objective Function}

Formally, we seek to minimize the annual energy use of the system $f(X)$. The "annual energy use" is taken as a weighted average of the system capacity at each of the 9 operating conditions, the weights being applied in accordance with the relative proportion of the prevailing climatic conditions. The system capacity is a function of the capacity (thermal output), of the air conditioning components, together with the fan power required to move air through the duct system.

\subsubsection{Constraint Functions and Infeasibility Function}

There are three sets of problem constraints, those concerned with the viability of the system topology, those with the procedure for evaluating the system performance and thirdly, those concerned with the system operation. The topology constraints are concerned with ensuring that that the system graph is connected in a manner that enables feasible operation. Each topology constraint is represented by an equality constraint (a binary constraint with a value $\in(0,1)$ ). The aggregated value of topology constraint violations $c_{\text {top }}\left(X_{d}\right)$ has been taken as the average of all topology constraint violations (the aggregated value then being in the range 0.0 to 1.0$)$.

The system performance evaluation constraints are concerned with identifying failure in the evaluation of system performance (in effect, failure to solve the system algebraic equations). The system operating constraints are concerned with ensuring that all components operate within their performance limits and that the system is able to maintain the desired zone conditions (the zone constraints being on the amount of outside air entering the zone and the zone temperature and humidity). The operation constraints are in the form of both inequality and equality constraints, although a reasonable engineering tolerance has been applied to the equality constraints. As in the case of the topology constraints, the constraints relating to the evaluation of system performance and to system operation, have been aggregated to have a value in the range 0.0 to 1.0 ( 0.0 indicating complete feasibility).

Note that, although there are three groups of constraints (topology constraints, performance evaluation constraints, system operation constraints), the system performance can only be evaluated if a feasible topology exists. Similarly, the system operation can only be evaluated if the system performance evaluation has been completed successfully. The constraint space is therefore discontinuous; this has been reflected in the formulation of an infeasibility function. If $c_{t o p}\left(X_{d}\right), c_{e v}(X)$ and $c_{o p}(X)$ are the aggregated constraint values for the topology, system performance evaluation, and system operating constraints respectively, then the system infeasibility $i(X)$, is given by:

$$
i(X)= \begin{cases}0.9+0.1 c_{\text {top }}\left(X_{d}\right), & \text { if }\left(c_{\text {top }}\left(X_{d}\right)>0.0\right) \\ 0.45+0.45 c_{e v}(X), & \text { else if }\left(c_{e v}(X)>0.0\right) \\ 0.45 c_{o p}(X), & \text { else }\end{cases}
$$

This gives three bands of infeasibility, infeasible topologies result in an infeasibility value in the range 0.9 to 1.0 , failure to evaluate the system performance, a value in the range 0.45 to 0.9 , and infeasible system operation in the range $>0.0$ to 0.45 . The choice 
of banding is arbitrary and its impact on behavior of the optimization has not been studied.

Note also, that the constraints on system performance evaluation and system operation are a function of all variables (albeit an implicit function of the topology variables), whereas the topology constraints are only a function of the topology variables.

\subsection{The Evolutionary Algorithm}

The general form of the evolutionary algorithm used here has been described previously [1,7]. In brief, a multi-chromosome genetic algorithm has been developed to solve the topological optimization problem, with each chromosome having its own distinct recombination and mutation operators. Ten chromosomes are active in the experiments reported here, one integer vector chromosome representing the system topology (system graph), and nine real vector chromosomes, one for each of the nine system operating (boundary) conditions.

Several new recombination and mutation operators have been developed for use with the topology chromosome, the goal of these operators being to perform effective recombination and mutation, while attempting to maintain the feasibility of the system topology. The operators and their effectiveness have been described previously [1]. In general, standard recombination and mutation operators were adopted for use with the real vector chromosomes, although some problem specific operators have been developed for use in combination with a "hyper-operation" (where the specific operator used at a given instance is decided probabilistically) [7].

The fitness assignment is based on the stochastic ranking algorithm [5], and selection by a binary tournament. A percentage of the best solutions are treated as elite individuals and copied directly to the next generation.

The algorithm control parameters used in the experiments reported here are given in Table 1. The effect of the algorithm control parameters on search performance has not been studied in depth, although preliminary experiments where conducted to confirm that the choice of parameters resulted in acceptable performance of the algorithm. In particular, although it is common to use high crossover rates in topological optimization [3], a $100 \%$ probability of crossover for the topology chromosome was found to be too disruptive, and resulted in a high probability of generating infeasible topologies; the same behavior was observed for the high mutation rates with the result that both the crossover and mutation rates for the topology chromosome are lower than used in solution of similar optimization problems. The ageing control parameter relates to the new algorithm operator.

\subsection{Topology Dominance}

Previously reported results indicated that the evolutionary algorithm had great potential to synthesize novel HVAC systems [1]. An analysis of the performance of the approach was subsequently extended to compare the energy use of the synthesized systems against that of two conventional systems [7] (referred to here as the "benchmark systems"). Many established HVAC system configurations exist, with the choice of which system is selected for a particular application depending on the cost constraints on building construction and operation. In this respect, the first of the benchmark systems is one with a moderate capital cost, but potentially higher than necessary operating cost
Table 1. Algorithm Control Parameters

\begin{tabular}{|c|c|c|}
\hline Operation & Control Parameter & Value \\
\hline \multirow{3}{*}{ Population } & Population Size & 1,000 \\
\hline & Maximum Generations & 10,000 \\
\hline & Elite Percentage & $2 \%$ \\
\hline $\begin{array}{l}\text { Tournament } \\
\text { Selection }\end{array}$ & Number of Individuals & 2 \\
\hline \multirow{2}{*}{ Recombination } & $\begin{array}{l}\text { Topology } \\
\text { Chromosome } \\
\text { Probability }\end{array}$ & $50 \%$ \\
\hline & $\begin{array}{l}\text { Operation } \\
\text { Chromosome } \\
\text { Probability }\end{array}$ & $100 \%$ \\
\hline \multirow{2}{*}{ Mutation } & $\begin{array}{l}\text { Topology } \\
\text { Chromosome } \\
\text { Probability }\end{array}$ & $2 \%$ \\
\hline & $\begin{array}{l}\text { Operation } \\
\text { Chromosome } \\
\text { Probability }\end{array}$ & $10 \%$ \\
\hline Stochastic Ranking & $\begin{array}{l}\text { Probability of } \\
\text { Infeasible Solution } \\
\text { Ranking }\end{array}$ & $45 \%$ \\
\hline ageing & $\begin{array}{l}\text { Maximum Evaluations } \\
\text { per Generation }\end{array}$ & 20 \\
\hline
\end{tabular}

(energy use); the second system has the higher capital cost, but for the example building studied, has the lower operating cost (energy use). In comparison to the performance of the two benchmark systems, the evolutionary algorithm was able to synthesize systems with an energy use in the range of the two benchmark systems. Although this represents a significant achievement in that the established systems are the result of over a century of engineering research and development, a thermodynamic analysis of the benchmark systems [7], suggested that they were less than optimal in terms of their energy use.

An analysis of the algorithms behavior in respect to the exploration of the topologies, indicated that at a single topology could become dominant at an early stage in the search. Figure 1, illustrates the life-span of every topology synthesized during a particular search. Each circle in the figure represents a unique topology, with the horizontal axis indicating the generation in which it was first synthesized. The vertical axis indicates the lifespan of the topology in terms of the number of times it appeared in any generation through the search (the total number of times it was evaluated). Clearly, the search illustrated in Figure 1 has been dominated by a single topology that first appeared at (approximately) the $1200^{\text {th }}$ generation, and was subsequently evaluated more than $7 \times 10^{6}$ times throughout the duration of the search. The dominance was further indicated by the topology accounting for up to $80 \%$ of the individuals in a given generation.

It was also concluded that the reason for the dominance was the implicit dependence of the system performance evaluation and operating constraints on the system topology. For instance, consider a solution which is completely feasible and therefore is likely to have a reasonably high fitness. If the effect of a mutation 
operation on the topology is to move the position of one of the components in the system, then it is highly likely that the solution will become infeasible as the system operation will no longer be valid (the change in position of the component would require a change in the capacity of one or more components if the system operation was to remain valid). In particular, the equality constraints on the condition of the zone air have a high probability of becoming infeasible when the system topology is changed.

Figure 1. Topology Dominance

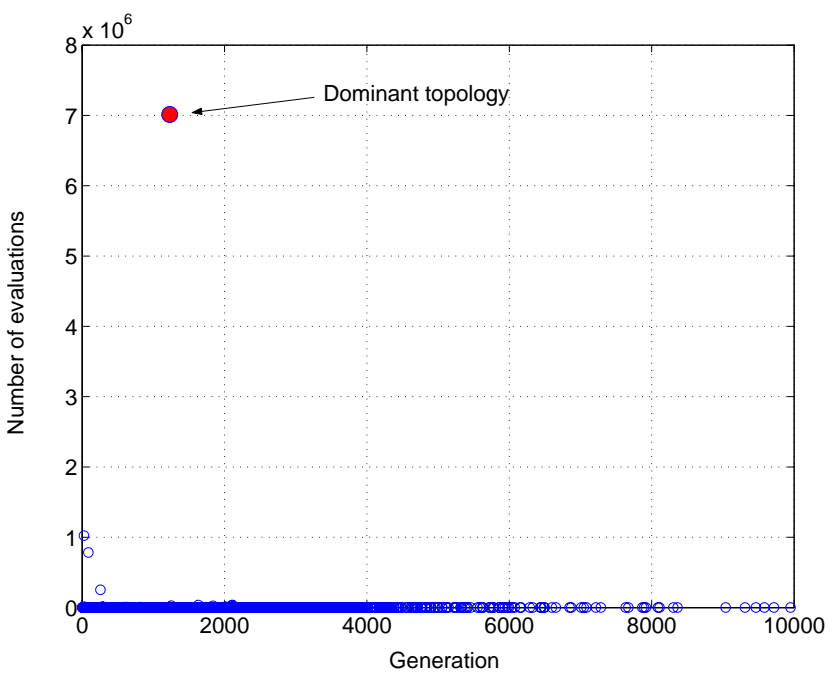

\section{AN AGEING OPERATOR}

The characteristic dominance of the search by a single topology (Figure 1), is a clear indication that the exploratory power of the search is limited. Such dominance can be addressed in several ways; the population could be "partitioned" such that a number of different topologies where forced to exist; or the spread of topologies could be maintained by a sharing function with the niche count derived from the topology chromosome only. Partitioning the population is likely to have a limited effect as the number of viable partitions and therefore alternative topologies is restricted by the population size. Further, the notion of "distance" between the different topologies as represented by the system graph (topology chromosome), is not clearly defined and although the topology chromosome has been designed to limit the duplicate representation of alternative topologies by numerically different chromosomes, the duplication is not guaranteed. As a result, a new algorithm operator, known as "ageing", was developed to prevent the dominance of the search by any one topology.

The ageing concept is that any one topology has a maximum number of evaluations, after which its fitness is reduced (as in real life, fitness declines with age). The life-span of the topology is defined here in terms of the number of times the topology is evaluated, rather than the number of generations that it has survived. This allows a topology to survive many generations provided that it does not dominate the population. The ageing function is given by:

$$
j^{\prime}(X)=j(X) \times \max \left[\left(n_{e}-q \times n_{g}\right), 1.0\right]
$$

where, $j(X)$ is the fitness of the individual, $j^{\prime}(X)$ its fitness after ageing, $n_{e}$ the number of times the topology has been evaluated, $q$, the maximum number of evaluations per generation allowed for a given topology, and $n_{g}$ the current generation number (number of generations to date).

The effect of the function is that provided the number of evaluations to date $\left(n_{e}\right)$, is less than the maximum allowed by the current generation $\left(q \times n_{g}\right)$, the fitness remains unchanged. If however, the number of evaluations exceeds the maximum allowed, then the fitness function value is increased by a factor equal to the number of evaluations in excess of the limit $\left(n_{e}-q \times n_{g}\right)$. Note that in this formulation, the higher the fitness, the lower the function value $(j(X))$, with the best solution having a function value of 0.0 . Since the best solution has a raw fitness of 0.0 , it is unaffected by the ageing operator, although any other individual in the population having the same topology would be subject to ageing. This acts to preserve at least one copy of the current best topology, while limiting its dominance (the best individual is preserved since we operate with a rank ordered population, with the top $2 \%$ of individuals being copiedto the new population).

Figure 2, illustrates the effect of the ageing operator on limiting topology dominance. As for Figure 1, the circles represent unique topologies, the horizontal axis the generation in which they appeared, and the vertical axis, the number of times a given topology was evaluated over the search period. The dashed diagonal line represents the ageing limit (in this case being 20 evaluations per generation, or $2 \%$ of the population per generation). Topologies with evaluations above the line have been subject to ageing, whereas those below survived without being aged.

Figure 2. Effect of Ageing on Topology Dominance

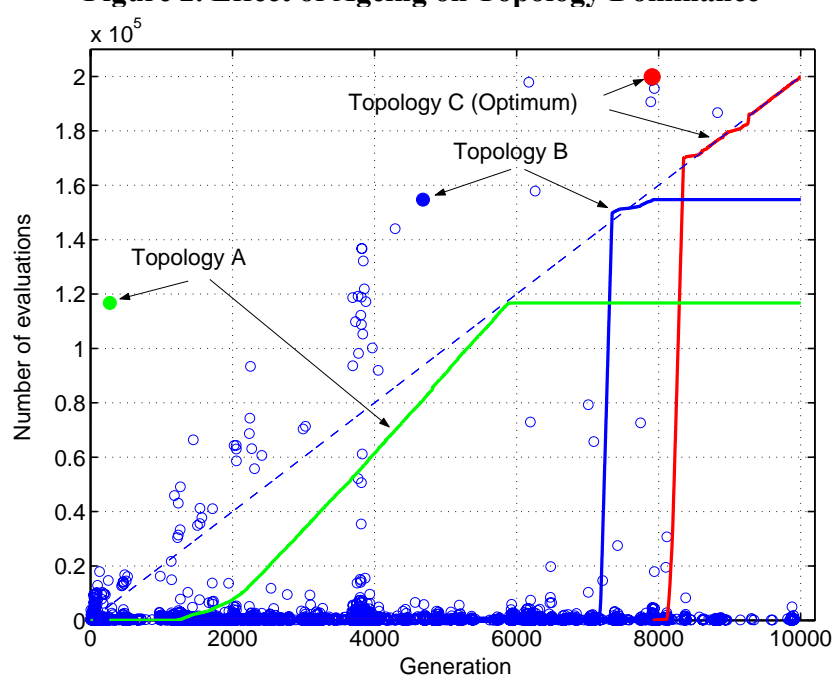

The three solid lines show the growth in number of evaluations for three of the topologies ('A', 'B' and 'C'). The point at which the growth line crosses the ageing limit indicates the generation at which ageing was applied to the particular topology. The three example topologies exhibit very different behavior in this respect. 
Topology ' $A$ ' appeared early in the search and since the number of duplicate topologies in any given population remained relatively low, the topology survived many generations before ageing was applied. Once aged, however, further growth in the number of evaluations was highly attenuated (on the scale of Figure 2, it would appear that there is no growth, although the topology may in fact continue to survive with a few copies in each subsequent generation).

Topology 'B' first appeared in mid-search, and remained "dormant" for many generations, until it experienced a rapid growth in the number of evaluations and subsequent application of ageing. The behavior of topology ' $\mathrm{C}$ ' is somewhat different to that of topology ' $A$ ' and ' $B$ ', in that the rapid growth in number of evaluations occurred soon after the topology first appeared. Further, unlike topologies ' $A$ ' and ' $B$ ', topology ' $C$ ' remained active after ageing, with the number of duplicate topologies appearing in each subsequent generation being in the order of the $2 \%$ of the population (equivalent to the ageing limit).

Figure 2, therefore illustrates that the ageing operator allows a topology to survive provided that it does not dominate the search, and that the number of topologies having a significant impact on the search has been increased (from 1 topology in Figure 1, to many in Figure 2).

Figure 3. Dynamic Effect of Ageing on the Population Diversity

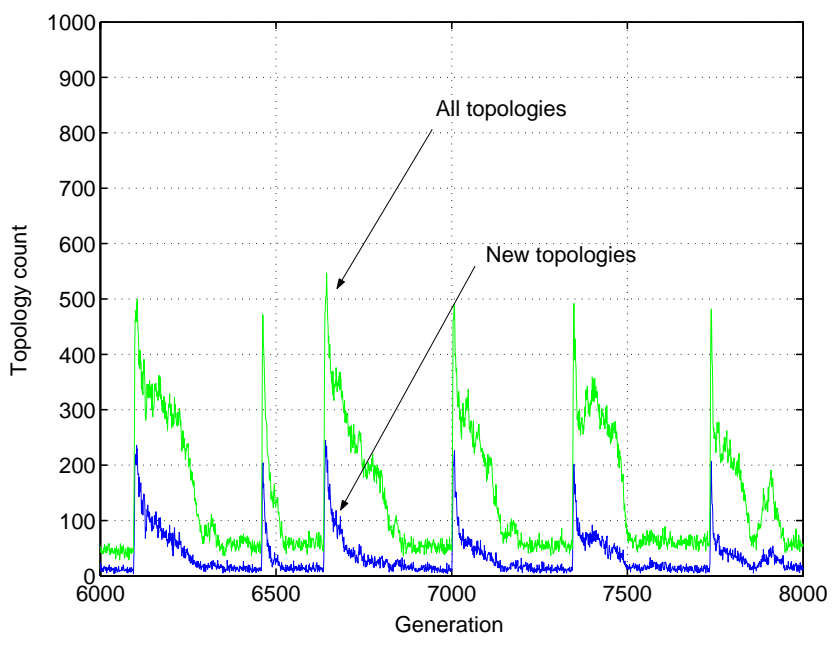

Figure 3, illustrates the dynamic effect of ageing on the population diversity (the vertical axis being the number of different topologies in a given generation). Each increase in the number of topologies corresponds to the application of the ageing operator to a dominant topology. As the fitness of the dominant topology is penalized, the diversity of the population grows both in terms of new and previously evaluated topologies.

A detailed analysis of the effect of ageing on the behavior of the search, or the effect of the ageing parameter $q$, has not been conducted, however, it can be concluded that, the ageing operator:

- $\quad$ prevents the long term dominance of the search by any one topology;

- allows topologies that have a low growth in number of evaluations to survive for many generations, whereas topologies that have a rapid increase in dominance of the population are prevented from prolonged dominance;

- and exhibits a dynamic behavior in the re-seeding of the population with new and previously searched topologies.

\section{EXPERIMENTS}

Two experiments have been conducted for the same example problem, one experiment on the performance of the algorithm without ageing, and second experiment with ageing.

\subsection{Example Problem and Benchmark Systems}

The example HVAC optimization problem is for a two zone building [7]. That is, the HVAC system is required to simultaneously conditions two separate spaces in the building. The level of difficultly associated with this "multi-zone" problem is significantly greater than for the design of a system that serves only one zone. The difficulty is associated with designing a system that can condition zones that may be experiencing different thermal loads and therefore have different conditioning needs. For example, it is possible that one zone will require cooling while a separate zone, conditioned by the same system, may require heating. In the example developed here, the operation of the system is optimized for 9 different boundary (load) conditions, within which 5 different system operating regimes occur (Table 2). As well as requiring the use of mechanical heating and cooling, under some boundary conditions, the system should be able to condition one or more zones through the use of moderately cool outside air alone (which is referred to as "free cooling" in Table 2).

Table 2. System Operating Regimes

\begin{tabular}{|c|c|}
\hline \multicolumn{2}{|c|}{ Zone } \\
\hline East & West \\
\hline \hline Heating & Heating \\
\hline Heating & Cooling \\
\hline Heating & Free cooling \\
\hline Free cooling & Free cooling \\
\hline Cooling & Cooling \\
\hline
\end{tabular}

\subsubsection{Problem Dimension}

The component set selected for the experiments resulted in 21 discrete variables encoded in the integer topology chromosome [7]. The number of alternative topologies resulting from the chromosome structure is $O(n !)$, which gives a search space of $5.1 \times 10^{20}$ topologies (although it is recognized that there is some duplication in the representation of the topologies and so the number of unique topologies is $<O(n !))$.

Each of the 9 boundary conditions results in 11 continuous operational variables, which gives a total of 99 continuous variables, encoded as 9 separate real chromosomes. Note that separate recombination and mutation operators are applied to each chromosome.

There are 10 equality constraints on the feasibility of the topology; 1 equality and 9 inequality constraints on the evaluation 
of system performance; and 108 inequality constraints on the operation of the system components (these being on the lowest temperature leaving the cooling coils and humidifiers, together with a restriction on the supply air temperature and air flow rates entering the zones; each of these being applied to all 9 boundary conditions).

More significantly, the quantity of outside air entering each zone is constrained by an inequality constraint, and the temperature and humidity of the air in each zone by equality constraints (each being subject to a tolerance). The zone constraints are applied for each boundary condition, so with 9 boundary conditions, and two zones, there are a total of 54 zone constraints (18 inequality and 36 equality constraints). The 36 equality constraints on the zone temperature and humidity are particularly difficult to solve.

In summary, there are a total of 120 problem variables, (21 discrete, and 99 continuous), and the problem is constrained by 135 inequality constraints and 55 equality constraints (although several of the equality constraints are subject to a tolerance, and many constraints are easily solved).

\subsubsection{Benchmark HVAC Systems}

Two established HVAC systems have been selected as benchmarks against which the performance of the synthesized systems will be judged. The systems are illustrated in Figures 4 and 5, in which components labeled with a $(+)$ are heating "coils", those with a (-) cooling coils. The components labeled with three "tear-drops" are steam humidifiers and the components forming junctions in the configuration represent sections of air duct used to merge or split the air-stream. Finally, the gray shaded shading of components indicates that for the example building, the components are not used for any load condition when the system operation has been optimized. This notation also applies to Figure 6.

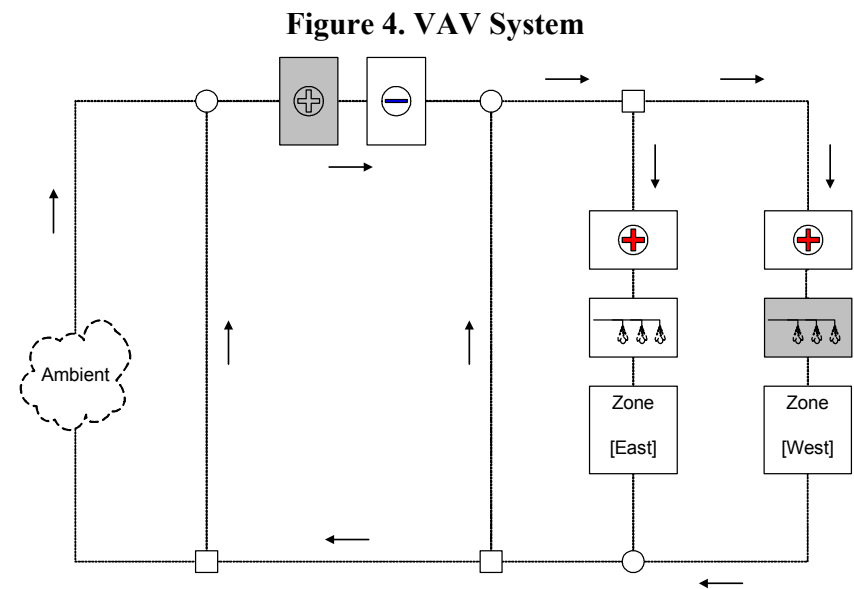

The first benchmark system is most commonly referred to as a "VAV system" (Figure 4) [2,7]. In brief, a VAV system has centralized heating and cooling components that performs the principal conditioning of the outside air. A conflict in load conditions, for instance one zone requiring heating while the other requires cooling, is handled by re-heating components located in the supply ducts to the individual zones. The zone humidity can also be controlled by steam humidifiers located in the supply ducts. Note that the operation of the benchmark systems has been optimized so that the differences in energy use between systems can be attributed to the effect of the system configuration alone and not the manner in which the system is controlled.

Figure 5, illustrates the second benchmark system, which is known as a "dual-duct" system. The dual-duct system differs from the VAV system in that the air supplied to a given zone can be supplied directly from either a "hot" duct, or a "cold" duct (containing hot and cold air respectively), or through a mix of air from both ducts. In the case of the two zone example, the dualduct system has a potentially lower energy use than the VAV system. In the case of the VAV system, when there is a conflict in the need for heating and cooling of the two zones, the air in the VAV system must be cooled in the centralized cooling coil only to then be re-heated before entering the heated zone. However, this is not necessary in the case of the dual-duct system, as cooling can take place in both the central duct and cold duct, so that conflicting loads can be met by simply supplying air from either duct. However, in comparison to the VAV system the extra ductwork of the dual-duct system is likely to increase the capital cost of the dual-duct system over that of the VAV system.

Figure 5. Dual-duct System

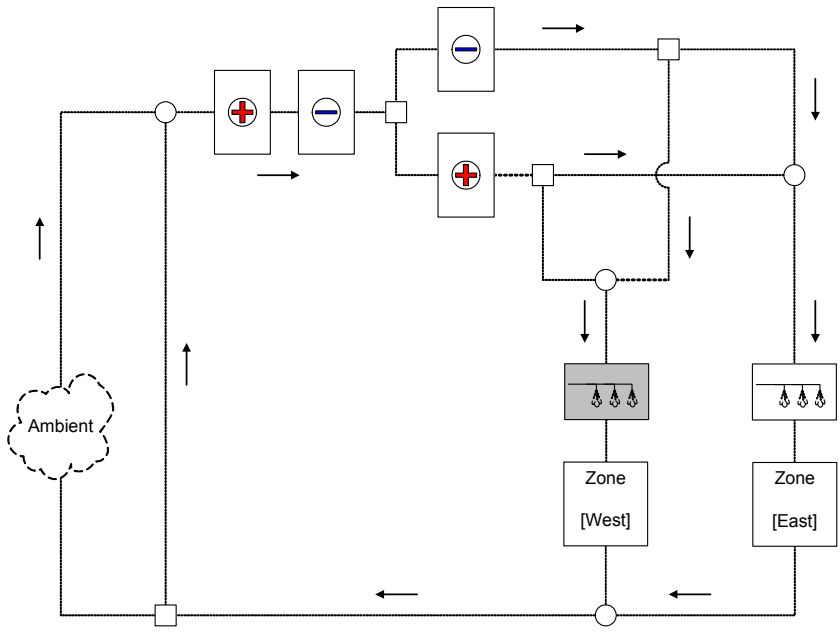

\subsection{Results}

The optimized energy use for the VAV and dual-duct benchmark systems was $7.42 \mathrm{~kW}$ and $5.13 \mathrm{~kW}$ respectively (energy use being represented by an averaged system capacity). Table 3 gives the results for the optimization. Without the use of ageing, only $28 \%$ of the trial optimizations resulted in a feasible solution. Ageing increased the rate of finding a feasible solution to $66 \%$. On inspection of the results, the principal cause of infeasibility in both experiments, appears to be the equality constraints on the zone temperature and humidity. The equality constraints on the system topology and system operation appeared less difficult to solve (the probability of a algorithm operator producing an infeasible topology being reduced by the use of problem specific recombination and mutation operators [1]).

In addition, the mean of the feasible solutions found without ageing has a higher energy use than the worst of the benchmark systems (the VAV system). The best solution found without ageing has an energy use that lies between that of the two benchmark systems. However, not only does the application of ageing lead to a mean energy use of the feasible solutions that lies between that of the two benchmark systems, but the best solution 
found has an energy use that is $15 \%$ lower than that of the best of the benchmark systems (the dual-duct system). The difference in the two means and corresponding variance has been used to confirm that there is greater than $95 \%$ confidence that there is a difference in the two means.

Table 3. Experimental Results

\begin{tabular}{|l||c|c|c|c|}
\hline \multirow{2}{*}{ Experiment } & \multirow{2}{*}{$\begin{array}{c}\text { Number } \\
\text { of Trials } \\
(-)\end{array}$} & $\begin{array}{c}\text { Percentage } \\
\text { of } \\
\text { Feasible } \\
\text { Solutions } \\
(\%)\end{array}$ & \multicolumn{2}{|c|}{$\begin{array}{c}\text { Feasible } \\
\text { Solutions }\end{array}$} \\
\cline { 4 - 5 } & & Mean & Best \\
$(\mathbf{k W})$ & $(\mathbf{k W})$ \\
\hline \hline $\begin{array}{l}\text { Without } \\
\text { ageing }\end{array}$ & 26 & 28 & 8.28 & 7.04 \\
\hline With ageing & 41 & 66 & 6.67 & 4.34 \\
\hline
\end{tabular}

Finally, the effectiveness of the ageing operator in increasing the exploratory power of the search is indicated by an order of magnitude increase in the number of topologies explored by the search with ageing in comparison to the number explored without ageing (Table 4).

Table 4. Effect of Ageing in Topology Exploration

\begin{tabular}{|l||c|}
\hline Experiment & $\begin{array}{c}\text { Mean Number } \\
\text { of Topologies } \\
\text { Explored } \\
\text { (-) }\end{array}$ \\
\hline $\begin{array}{l}\text { Without } \\
\text { ageing }\end{array}$ & $9.45 \times 10^{4}$ \\
\hline With ageing & $6.33 \times 10^{5}$ \\
\hline
\end{tabular}

\subsubsection{Best of the Synthesized Systems}

The best of the optimized system configurations is illustrated in Figure 6. The synthesized system has a completely new configuration having several novel features. First, note that each zone is conditioned by a separate set of components, which enables the system to have a separate response to the thermal loads in each zone. In many systems, such as the VAV system (Figure 4), conditioned air is cooled centrally only to be re-heated before being supplied to a zones requiring less cooling than others (in the worst case, some zones will require heating rather than cooling).

A second novel feature of the optimized system, is that it capitalizes on the potential to transfer energy between the different zones in the building. In this case, the air leaving the east zone passes through the west zone before being exhausted to atmosphere; this does not take place in conventional systems (Figure 4, and 5). For the example building, one result of the inter-zone air-flow is that the need for steam humidifiers has been eliminated, whereas the west zone required humidification by the VAV and dual duct systems (compare the gray shaded components in Figures 4, 5, and 6, which indicates that they are never operated).

Finally, a novel feature not found in any other HVAC system, is that the components associated with the west zone have been placed in the air ducts that have the lower air-flow rates. The lower the air-flow rate across a component, the lower the airpressure loss it generates and the lower the associated fan energy use. The air-flow rates in the air re-circulation legs of the duct system are lower than in the main duct sections (the sections after the merging of two air-streams). This effect is most noticeable for boundary conditions when no mechanical heating or cooling is required (the "free cooling" operation in Table 2). Under these conditions, the re-circulation air-flow is greatly reduced and may be zero (the effect of a zero air-flow rate being that there would be no fan energy use associated with components). The fact that the components associated with the east zone have not been moved to a re-ciculation leg of the duct system indicates that, although the system has a $15 \%$ lower energy use than the best of the benchmark systems, the synthesized system is slightly suboptimal.

Figure 6. Best Synthesized System

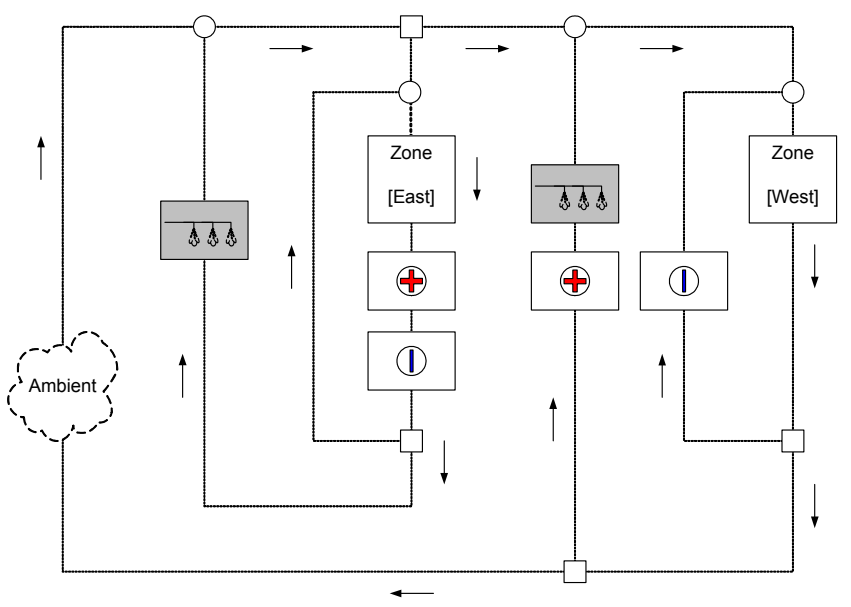

\section{CONCLUSIONS}

The topological design of HVAC systems is a highly constrained, multi-modal optimization problem. Experiments indicated that a multi-chromosome evolutionary algorithm with problem specific operators for the recombination and mutation of the system topology chromosomes [1], was able to synthesis systems having an energy use comparable to some established systems. However, the performance of the synthesized systems remained low in comparison to the most energy efficient of the established systems. It was observed that the cause of sub-optimality of the solutions was due to the dominance of the search by a single topology. The dominance was due to the equality constraints on the HVAC system operation, a change in topology having a high probability that a previously feasible solution would violate one or more of the equality constraints.

A new evolutionary algorithm operator was introduced to inhibit the dominance of the search by a single topology. The "ageing" operator functions by penalizing the fitness of solutions which have a dominant topology, the dominance of the topology being measured by the number of times the topology has been evaluated at a given point in the search. The ageing penalty is only applied when the number of function evaluations exceed a limit defined in terms of a maximum number of function evaluations per generation. Hence, topologies that occupy the population by less than the maximum number per generation are not penalized, 
whereas those that exceed the limit are penalized. It was concluded that the ageing operator:

- $\quad$ prevents the long term dominance of the search by any one topology;

- $\quad$ exhibits a variety of different dynamic behaviors in terms of the growth in dominance and its penalization; these range from long slow growth and weak penalization over many generations, to rapid growth in dominance with a correspondingly severe penalization of solutions;

- and exhibits a dynamic behavior in the re-seeding of the population with new and previously searched topologies;

- $\quad$ increases the number of topologies searched;

- requires further research to investigate the effect of the ageing limit parameter on the dynamic behavior of the search.

It was demonstrated that the application of the ageing operator increases the probability of finding a feasible solution from $28 \%$ to $66 \%$ (the example problem being highly constrained). It was concluded that the cause of infeasibility was due to the search being unable to solve one or more of the equality constraints on the zone temperature and humidity. Given that these constraints are dependent on the boundary conditions on the system operation, and that the system operation is represented by a separate chromosome for each boundary condition, it is concluded that the effectiveness of the algorithm may be improved through the application of chromosome specific selection and recombination, the selection and recombination being a function of sub-fitness at a particular boundary condition (in a similar manner to that reported in $[4,6])$.

It was also demonstrated that the introduction of the ageing operator, resulted in a statistically significant improvement in the feasible objective function values found by the evolutionary algorithm. In particular, the system configuration for the best solution found when using the ageing operator, had novel features that enabled it to operate with a $15 \%$ lower energy use than the best of the conventional systems (this being considered a significant achievement, as the established systems are a result of over a century of engineering research and development).

It can be concluded that the ageing operator had a significant impact on the performance of the evolutionary algorithm in solving this highly constrained topological optimization problem, and the best solution found by the search represented a novel design having a significantly better performance than established system designs.

\section{ACKNOWLEDGMENTS}

This work was funded by the American Society of Heating, Refrigerating, and Air-Conditioning Engineers (ASHRAE), under research project RP-1049.

\section{REFERENCES}

[1] Angelov, P.P., Zhang. Y., Wright, J.A., Hanby, V.I., and Buswell, R.A., Automatic Design Synthesis and Optimization of Component-Based Systems by Evolutionary Algorithms, In GECCO 2003, Lecture Notes in Computer Science 2724, Cantu-Paz et al. (Eds), 1938-1950, 2003.

[2] ASHRAE, HVAC Systems and Equipment, ASHRAE Handbook, ASHRAE, 1791 Tullie Circle, N.E. Atlanta, GA 30329, USA, ISBN 0-910110-87-5, 2000.

[3] Koza, J. R., Keane, M. A., Streeter, M. J., Mydlowec, Y. J., and Lanza, G, Genetic Programming IV: Routine HumanCompetitive Machine Intelligence, Kluwer Academic Publishers, ISBN 1-4020-7446-8, 2003.

[4] Loosemore, H.A., The Multi-Objective Optimum Design of Building Thermal Systems, PhD Thesis, Loughborough University, Loughborough, Leicestershire, LE11 3TU, UK, 2003.

[5] Runarsson, T.P., and Yao, X., Stochastic Ranking for Constrained Evolutionary Optimisation, IEEE Transactions on Evolutionary Computation, 4, 3, 284-294, 2000.

[6] Wright, J.A., Loosemore, H.A., Farmani, R., Optimization of Building Thermal Design and Control by Multi-Criterion Genetic Algorithm, Energy and Buildings, 34, 959-972, 2002.

[7] Wright, J.A., Zhang, Y., Angelov, P.P., Buswell, R.A., Hanby, V.I., Building System Design Synthesis and Optimization (Final Report, ASHRAE RP-1049), Available from ASHRAE, 1791 Tullie Circle, N.E. Atlanta, GA 30329, USA, 2004. 